\title{
Combination therapy of transcatheter arterial chemoembolization with axitinib for the treatment of inoperable hepatocellular carcinoma
}

\author{
Kazuhiro Nouso, Akiko Wakuta, Kazuya Kariyama \\ Department of Gastroenterology, Okayama City Hospital, Okayama, Japan \\ Correspondence to: Kazuhiro Nouso, MD, PhD. Department of Gastroenterology, Okayama City Hospital, 3-20-1 Kitanagase-omotemachi, Kita-ku, \\ Okayama 700-8557, Japan. Email: kazunouso@gmail.com. \\ Provenance and Peer Review: This is an invited article commissioned by the Editorial Office, Annals of Translational Medicine. The article did not \\ undergo external peer review. \\ Comment on: Chan SL, Yeo W, Mo F, et al. A phase 2 study of the efficacy and biomarker on the combination of transarterial chemoembolization and \\ axitinib in the treatment of inoperable hepatocellular carcinoma. Cancer 2017;123:3977-85. \\ Kudo M, Ueshima K, Ikeda M, et al. Randomised, multicentre prospective trial of transarterial chemoembolisation (TACE) plus sorafenib as \\ compared with TACE alone in patients with hepatocellular carcinoma: TACTICS trial. Gut 2019. [Epub ahead of print].
}

Submitted Mar 08, 2020. Accepted for publication Mar 20, 2020.

doi: 10.21037/atm.2020.03.198

View this article at: http://dx.doi.org/10.21037/atm.2020.03.198

Transcatheter arterial chemoembolization (TACE) is a standard recommended therapy for the treatment of intermediate-stage hepatocellular carcinoma (HCC) (1). TACE obstructs tumor arteries and depletes blood supply, resulting in tumor death. However, recurrence at the treated site has often been observed because not all cancer cells were killed by TACE, even in cases with complete embolization. Therefore, TACE must be repeated in many cases. Although TACE can treat HCC effectively, it simultaneously damages liver function. Therefore, new strategies to increase the effectiveness of TACE are warranted. Thus, possible combination therapies with chemotherapeutic agents, especially with molecular target agents (MTAs), have been examined. The Post-TACE (2), SPACE (3), and TACE-2 (4) studies were randomized control trials (RCTs) that examined the additive effect of sorafenib on TACE. The BRISK-TA (5) and ORIENTAL (6) RCT studies examined the additive effects of brivanib and orantinib, respectively. These drugs were used mainly to suppress the effect of the angiogenic cytokine surge induced by hypoxia after TACE. The strategies were logical; however, the results of these studies were dismal.

Recently, the TACTICS trial (7) successfully showed the effectiveness of a combination therapy. This multicenter prospective RCT compared TACE/sorafenib combination with TACE alone and shed light on this field. A new combination therapy with axitinib was also reported by Chan et al. (8). This phase II, single-arm study examined the efficacy of TACE/axitinib combination in the treatment of inoperable HCC. The 2-year overall survival (OS) was $43.7 \%$, which was higher than the average 2-year OS for TACE monotherapy in that region $(31 \%)$. Interestingly, emergence of hypertension was closely related to better progression-free survival (PFS): 11.6 months in the presence of hypertension vs. 4.5 months in its absence $(\mathrm{P}=0.0017)$.

Axitinib, an inhibitor of vascular endothelial growth factor receptor, is approved as a second-line treatment for renal cell carcinoma (9). Although axitinib did not prolong OS compared to placebo in a randomized phase II trial for the treatment of advanced HCC, improvements in PFS and time to tumor progression were observed (10). In an exploratory analysis that excluded patients intolerant of prior antiangiogenic therapy, OS favored axitinib over placebo in Asia, especially in Japan (11). Thus, it might be reasonable to use axitinib in combination with TACE.

Several points are key to obtaining beneficial effects with combination therapies. Good therapeutic effects with TACE are better obtained by first injuring cancer cells prior to TACE. Injured cells can be susceptible to hypoxia induced by embolization of tumor-feeding arteries, which 
Table 1 Difference between TACE/axitinib combination therapy and TACTICS

\begin{tabular}{lll}
\hline Factors & TACE/axitinib combination therapy* & TACTICS $^{\star *}$ \\
\hline Treatment before TACE & 10 mg axitinib for 5 weeks before TACE & 400 mg sorafenib for 2-3 weeks before TACE \\
Treatment after TACE & Resume axitinib 24 hours after TACE & Resume sorafenib 3 days after TACE \\
Primary endpoint & 2-year OS rate & $\begin{array}{l}\text { OS and PFS; "new intrahepatic lesions were not regarded as } \\
\text { progressive disease" }\end{array}$ \\
\hline
\end{tabular}

*, reference (8); ${ }^{*}$, reference (7). TACE, transcatheter arterial chemoembolization; OS, overall survival; PFS, progression-free survival.

implies that the administration of chemotherapeutic agent before TACE may be a better strategy. Drug administration is usually stopped temporarily when commencing TACE, to prevent a synergistic adverse effect, then re-started afterward. It is an important point that early resumption of the drug is logically better, in terms of avoiding the effect of angiogenic cytokine surge induced by TACE. The Post-TACE study (2) was designed to delay the recurrence of HCC, and to prolong OS, by administering sorafenib after TACE. When the study failed, the late start of sorafenib (1-3 months after TACE) was listed as a major problem. Later, the TACTICS trial (7), which adjusted the timing of sorafenib treatment, successfully showed the combination treatment to have a beneficial effect, and so the timing of the administration must be an important consideration in planning new studies.

Timing was successful in the case of TACE/axitinib combination therapy, where TACE was planned to occur during a 56-day axitinib treatment period, with only a 48-hour discontinuation of axitinib at the time of TACE. One concern is the very long axitinib pre-treatment period of 5 weeks in each cycle. This was much longer than that in TACTICS (2-3 weeks), and it may have been too long to produce the effect of axitinib.

Primary endpoint is another key factor to properly establish the effect of combination therapy. OS has been considered to be a standard endpoint for evaluating the efficacy of anticancer drugs $(12,13)$. Because most patients with intermediate-stage HCC will receive poststudy therapies, including strong life-prolonging MTA, the efficacy of the combination therapy cannot be evaluated by OS. Among studies of TACE combination therapies, including the Post-TACE, BRISK-TA, SPACE, ORIENTAL, TACE2, and TACTICS studies, PFS was the primary endpoint used in half of the studies, and OS in the rest. All studies that adopted OS as their primary endpoint failed. The only study with a positive result, TACTICS, adopted a modified PFS as its endpoint.

Even though pre-existing lesions were well-controlled by TACE, new lesions usually emerged, which is the typical course of HCC after TACE. However, these patients were classified as Progressive Disease under the RECIST/modified RECIST criteria $(14,15)$, resulting in MTA treatment of insufficient duration. To overcome this problem, Kudo et al. ignored the emergence of new lesions for evaluating the effect of TACE/sorafenib combination therapy, and proved the benefit of the combination therapy (7). Although 2-year OS for the TACE/axitinib group was better than that reported for TACE in the same region, it would be preferable to use a different primary endpoint when considering experiences with TACE in earlier studies. The differences between TACE/axitinib combination study and TACTICS were summarized in Table 1.

As a matter of course, demonstrating benefit of combination therapies requires proper TACE procedure, proper selection of patients, and careful dose management in order to prolong the treatment period and deliver adequate drug effect. In the study of TACE/axitinib treatment, patients with emergence of hypertension due to axitinib showed better PFS. This means that emergence of hypertension may be useful to select good candidates for the next cycle of combination therapy (8). In addition, a close relationship was reported between OS of axitinib-treated patients with advanced HCC and serum markers such as microRNAs and cytokines $(10,11)$. These markers might also be useful for the combination treatment with axitinib/ TACE, although validation is needed.

Recently, many MTAs have been developed, and effective sequential therapies are being explored. The benefit of axitinib in combination with TACE must be re-evaluated with these new therapies. Nonetheless, the first step for each of the new therapies is to determine its efficacy, and the combination therapy of axitinib/TACE remains a strong candidate.

\section{Acknowledgments}

Funding: None. 


\section{Footnote}

Conflicts of Interest: All authors have completed the ICMJE uniform disclosure form (available at http://dx.doi. org/10.21037/atm.2020.03.198). The authors have no conflicts of interest to declare.

Ethical Statement: The authors are accountable for all aspects of the work in ensuring that questions related to the accuracy or integrity of any part of the work are appropriately investigated and resolved.

Open Access Statement: This is an Open Access article distributed in accordance with the Creative Commons Attribution-NonCommercial-NoDerivs 4.0 International License (CC BY-NC-ND 4.0), which permits the noncommercial replication and distribution of the article with the strict proviso that no changes or edits are made and the original work is properly cited (including links to both the formal publication through the relevant DOI and the license). See: https://creativecommons.org/licenses/by-nc-nd/4.0/.

\section{References}

1. European Association for the Study of the Liver. EASL Clinical Practice Guidelines: management of hepatocellular carcinoma. J Hepatol 2018;69:182-236.

2. Kudo M, Imanaka K, Chida N, et al. Phase III study of sorafenib after transarterial chemoembolisation in Japanese and Korean patients with unresectable hepatocellular carcinoma. Eur J Cancer 2011;47:2117-27.

3. Lencioni R, Llovet JM, Han G, et al. Sorafenib or placebo plus TACE with doxorubicin-eluting beads for intermediate stage HCC: the SPACE trial. J Hepatol 2016;64:1090-8.

4. Meyer T, Fox R, Ma YT, et al. Sorafenib in combination with transarterial chemoembolisation in patients with unresectable hepatocellular carcinoma (TACE 2): a randomised placebo-controlled, double-blind, phase 3 trial. Lancet Gastroenterol Hepatol 2017;2:565-75.

5. Kudo M, Han G, Finn RS, et al. Brivanib as adjuvant therapy to transarterial chemoembolization in patients with hepatocellular carcinoma: A randomized phase III

Cite this article as: Nouso K, Wakuta A, Kariyama K. Combination therapy of transcatheter arterial chemoembolization with axitinib for the treatment of inoperable hepatocellular carcinoma. Ann Transl Med 2020. doi: $10.21037 /$ atm.2020.03.198

(C) Annals of Translational Medicine. All rights reserved. trial. Hepatology 2014;60:1697-707.

6. Kudo M, Cheng AL, Park JW, et al. Orantinib versus placebo combined with transcatheter arterial chemoembolisation in patients with unresectable hepatocellular carcinoma (ORIENTAL): a randomised, double-blind, placebo-controlled, multicentre, phase 3 study. Lancet Gastroenterol Hepatol 2018;3:37-46.

7. Kudo M, Ueshima K, Ikeda M, et al. Randomised, multicentre prospective trial of transarterial chemoembolisation (TACE) plus sorafenib as compared with TACE alone in patients with hepatocellular carcinoma: TACTICS trial. Gut 2019. [Epub ahead of print].

8. Chan SL, Yeo W, Mo F, et al. A phase 2 study of the efficacy and biomarker on the combination of transarterial chemoembolization and axitinib in the treatment of inoperable hepatocellular carcinoma. Cancer 2017;123:3977-85.

9. Rini BI, Escudier B, Tomczak P, et al. Comparative effectiveness of axitinib versus sorafenib in advanced renal cell carcinoma (AXIS): a randomised phase 3 trial. Lancet 2011;378:1931-9.

10. Kang YK, Yau T, Park JW, et al. Randomized phase II study of axitinib versus placebo plus best supportive care in second-line treatment of advanced hepatocellular carcinoma. Ann Oncol 2015;26:2457-63.

11. Kudo M, Kang YK, Park JW, et al. Regional differences in efficacy, safety, and biomarkers for second-line axitinib in patients with advanced hepatocellular carcinoma: from a randomized phase II study. Liver Cancer 2018;7:148-64.

12. Llovet JM, Di Bisceglie AM, Bruix J, et al. Design and endpoints of clinical trials in hepatocellular carcinoma. J Natl Cancer Inst 2008;100:698-711.

13. Llovet JM, Zucman-Rossi J, Pikarsky E, et al. Hepatocellular carcinoma. Nat Rev Dis Primers 2016;2:16018.

14. Eisenhauer EA, Therasse P, Bogaerts J, et al. New response evaluation criteria in solid tumours: revised RECIST guideline (version 1.1). Eur J Cancer 2009;45:228-47.

15. Lencioni R, Llovet JM. Modified RECIST (mRECIST) assessment for hepatocellular carcinoma. Semin Liver Dis 2010;30:52-60. 